\section{ECCOMAS}

Proceedia
COMPDYN 2019

$7^{\text {th }}$ ECCOMAS Thematic Conference on Computational Methods in Structural Dynamics and Earthquake Engineering M. Papadrakakis, M. Fragiadakis (eds.) Crete, Greece, 24-26 June 2019

\title{
NUMERICAL EVALUATION OF THE MODAL CHARACTERISTICS OF A BRIDGE ABUTMENT
}

\author{
Davide N. Gorini ${ }^{1}$, Luigi Callisto ${ }^{1}$, and Andrew J. Whittle ${ }^{2}$ \\ ${ }^{1}$ Sapienza University of Rome \\ Via Eudossiana 18, Roma, Italy \\ e-mail: \{davideno.gorini,luigi.callisto\}@uniroma1.it \\ ${ }^{2}$ Massachusetts Institute of Technology \\ 77 Massachusetts Avenue, Cambridge (MA), United States \\ ajwhittl@mit.edu
}

\begin{abstract}
The seismic analysis of bridges needs to account for the effects of soil-structure interaction with methods that strike a reasonable balance between completeness and reliability of the numerical soil-structure interaction models. In view of this, focusing on the marked influence that the behaviour of the abutments can have on the seismic performance of the whole bridge, this study presents an identification procedure of the dynamic response bridge abutments. Based on the results of dynamic simulations on a reference local model of a bridge abutment carried out in the analysis framework OpenSees, the modal characteristics of the soilabutment system are computed and are used to evidence the role played by the soil interacting with the abutment in controlling the overall dynamic response of the system. This role is quantified through the definition of the mass participation factors for different directions of motion.
\end{abstract}

Keywords: bridge abutments, frequency-dependent response, inertial effects, OpenSees. 


\section{INTRODUCTION}

Seat-type abutments can influence significantly the seismic performance of a bridge, because of their interaction with the backfill and of the significant inertial forces developing during the earthquake. An evaluation of the dynamic effects of bridge abutments was proposed by Wissawapaisal [1], who regarded the embankment interacting with the abutment as an assembly of lumped masses connected through nonlinear shear springs. The calibration of this type of models depends on the evaluation of an effective length of embankment that participates to the dynamic response of the bridge. Kotsoglou and Pantazopoulou [2], following an analytical approach, provided solutions for the modal characteristics of the soil-abutment system in the transverse direction of the bridge, considering a simplified geometry of the abutment-embankment system under the assumption of linear behaviour of soil. The compliance of the soil beneath the abutment foundation was therein neglected with a consequent overestimation of the global stiffness of the abutment system.

The present study is part of a wider research project on the dynamic response of abutments aimed to identify simplified macro-element representations of the soil-abutment system. In this context, the present paper presents the evaluation of the dynamic properties of a reference bridge abutment, obtained through the dynamic analysis of an advanced numerical model of the soil-abutment system.

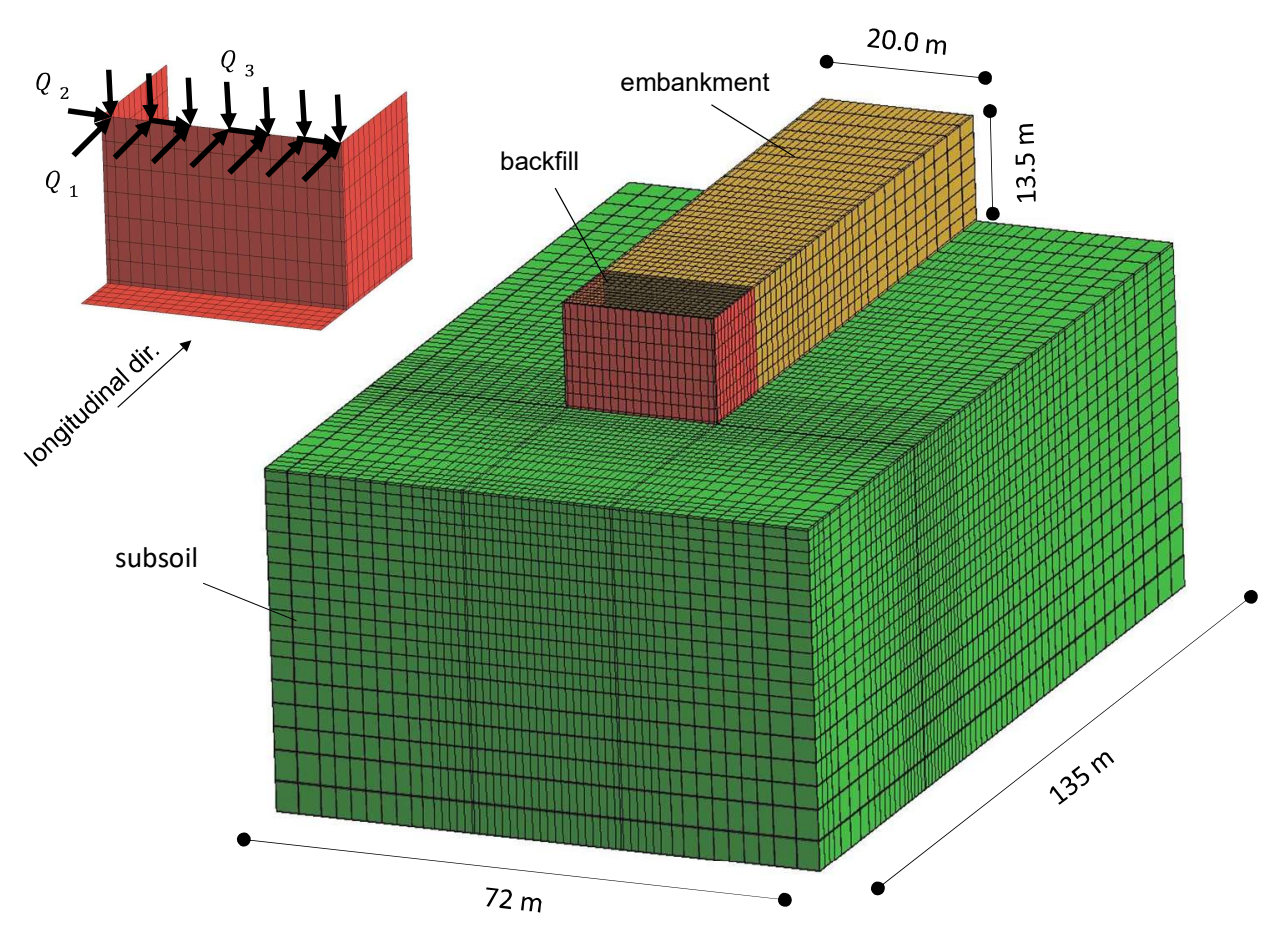

Figure 1: Soil-abutment interaction model implemented in OpenSees to study the frequency-dependent response of the reference abutment.

\section{REFERENCE CASE STUDY}

This study takes as a reference the soil-bridge system developed by Gorini and Callisto [3]. With the aim to investigate the dynamic response of the abutments, a local model of the soilabutment system was implemented in the open-source finite element analysis framework OpenSees [4], with mesh generation performed through the pre/post-processor software GID [6]. The model, shown in Figure 1, includes the abutment, the approach embankment and a homogeneous subsoil, representing the upper layer of the Messina Gravels [7]. The abutment 
is a massive reinforced concrete structure resting on a foundation slab. The central wall has a height of $13.5 \mathrm{~m}$ and a thickness of $4 \mathrm{~m}$, while the dimensions of the foundation are $17 \mathrm{~m}$ and $20 \mathrm{~m}$ in the longitudinal and transverse directions, respectively. All the structural members were modelled through the ShellMITC4 elements [9] with elastic behaviour, using constitutive parameters relative to a C32/40 strength class concrete in the European standards. A Rayleigh damping was assigned to the elements of the abutment, calibrated in order to produce a damping ratio not larger than $2 \%$ for all the significant modes of the abutment.

The entire soil domain was discretized through 49834 SSPbrick eight-noded hexahedral elements for a whole plan extension of $135.0 \times 72.0 \mathrm{~m}^{2}$. The horizontal extension of the model is equal to 3.6 and 7.7 times the transverse and longitudinal dimensions of the abutment, while the soil domain extends down to a depth of $60 \mathrm{~m}$ from the abutment foundation, that is 3 times the largest size of the foundation. Preliminary dynamic analyses indicated that the lateral boundaries were distant enough not to affect the computed behaviour of the abutment. The embankment behind the abutment wall was regarded as an equivalent single-phase material, with properties that account for typical suction levels. The mechanical behaviour of the soil was described with the Pressure Dependent Multi-Yield model (PDMY) developed by Yang et al. [10], calibrated against numerous experimental data under both monotonic and cyclic conditions. The constitutive parameters assigned to the foundation soil and the embankment are reported in Table 1, while the reader can refer to Gorini [11] for a detailed description of the calibration procedure. For simplicity, the soil domain was assumed dry.

\begin{tabular}{llcc}
\hline Variable & Description & Subsoil & Embankment \\
\hline$\rho\left(\mathrm{Mg} / \mathrm{m}^{3}\right)$ & mass density & 2.243 & 2.039 \\
$G_{\mathrm{r}}(\mathrm{kPa})$ & elastic shear modulus at $p_{\mathrm{r}}{ }^{\prime}$ & $1.3 \times 10^{5}$ & $1.5 \times 10^{5}$ \\
$v$ & Poisson's ratio & 0.2 & 0.2 \\
$p_{\mathrm{r}}{ }^{\prime}(\mathrm{kPa})$ & reference mean pressure & 80.0 & 80.0 \\
$d$ & pressure dependent coefficient & 0.5 & 0.5 \\
$\gamma_{\mathrm{d}, \max }$ & peak shear strain & 0.1 & 0.1 \\
$\varphi_{\mathrm{PTL}}$ & phase transformation angle & $26^{\circ}$ & $26^{\circ}$ \\
$c$ & contraction parameter & 0.195 & 0.195 \\
$d_{1}$ & dilation parameters & 0.6 & 0.6 \\
$d_{2}$ & critical stress ratio & 3.0 & 3.0 \\
$M$ & & 1.54 & 1.42 \\
$\lambda_{\mathrm{c}}$ & Critical State Line parameters & 0.02 & 0.02 \\
$e_{0}$ & & 0.9 & 0.9 \\
$\xi$ & number of yield surfaces & 0.7 & 0.7 \\
$N$ & & 40 & 40 \\
\hline
\end{tabular}

Table 1: Parameters of the PDMY model for the subsoil (Messina Gravels) and the embankment.

A staged analysis procedure was adopted, with an initial application of the gravity loads. The construction of the abutment and the embankment was simulated sequentially. The successive dynamic simulation was performed with the parallel computing using the OpenSeesSP interpreter [12]. The nodes at the base of the model were restrained in all directions while the lateral boundaries of the subsoil were allowed to displace in the vertical direction only; the nodes on the opposite lateral sides of the embankment were constrained to undergo the same motion as a simplified manner to simulate the confinement produced by the side slopes. 
The dynamic perturbation consisted of a distributed force line $Q_{\mathrm{i}}$ applied to the top of the central wall of the abutment, varying harmonically with a period $T$ for 10 loading cycles. The period $T$ ranged between $0.05 \div 5.0 \mathrm{~s}$. The force was applied separately for each load direction at the deck-abutment contact.

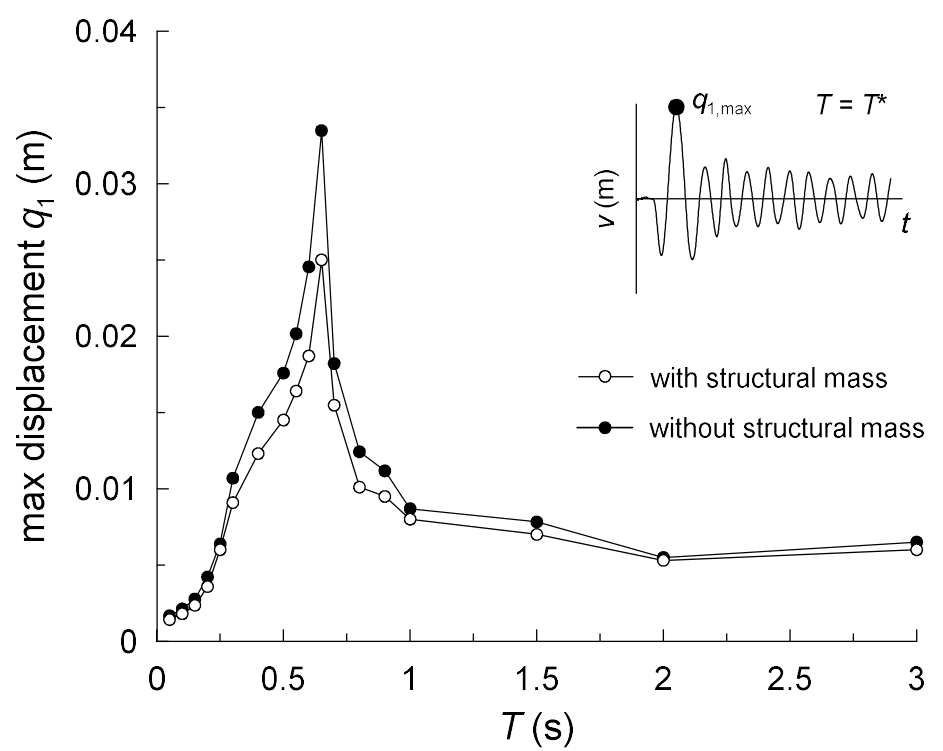

Figure 2: Dynamic amplification curve at small displacements of the soil-abutment system in the longitudinal direction of the deck-abutment contact.

\section{DYNAMIC IDENTIFICATION}

\subsection{Dominant periods}

As a first result, the dynamic identification of the system is limited to the reversible response of the abutment, considering a sufficiently small amplitude of the external force (in the present case $Q_{\mathrm{i}} \leq 1200 \mathrm{kN} / \mathrm{m}$ ) so that no significant permanent displacements occur at the deck-abutment contact during the analysis. Because the focus is on the soil-abutment interaction effects transmitted to the bridge structure, the dynamic response of the soil-abutment system is represented in terms of dynamic amplification curves that relate the maximum longitudinal displacement of the abutment top to the period of the correspondent external force. Figure 2 shows the amplification curve in the longitudinal direction: the maximum displacement does not increase monotonically as the period rises and a dominant peak is well defined at a period $T_{\mathrm{D} 0}{ }^{\text {long }}=0.6 \mathrm{~s}$ produced by the resonance of the soil-abutment system. The figure shows that the structural mass of the abutment has a minor effect on the response, leading to a modest increment of the displacement in the region of maximum amplification ( $T=0.4 \div 1 \mathrm{~s}$ ), without altering the dominant period of the system. Therefore, the dynamic amplification of the soil-abutment system seems to be controlled mainly by the participating mass of the soil interacting with the wall, that appears be significantly larger than the abutment mass.

In Figure 3, the longitudinal response of the abutment is compared with the amplification curves in the transverse and vertical directions. In all directions, the dynamic amplification concentrates between $T=0.2 \div 1.0 \mathrm{~s}$. More in detail, the vertical direction shows a dominant peak at $T_{\mathrm{D} 0}{ }^{\mathrm{vert}}=0.4 \mathrm{~s}$, leading to a stiffer response if compared to the horizontal modes of the deck-abutment contact. The transverse response follows quite closely the curve relative to the longitudinal direction but presents a slightly larger dominant period $T_{\mathrm{D} 0}{ }^{\operatorname{tran}}=0.7 \mathrm{~s}$. The longer 
dynamic response in the transverse direction might be due to the fact that, differently from the other directions, in the transverse direction there is no lateral confinement to the abutment wall and therefore the vibrations of the abutment are mainly controlled by the interaction with the foundation soil only, causing a slight increment of the deformability of the soil-abutment system. The fundamental period of the abutment structure alone, preliminarily evaluated through a modal analysis of the structure, is $T_{0}^{\text {abut }}=0.1 \mathrm{~s}$ and therefore is completely decoupled from the dynamic response of the whole system. Hence, it is reasonable to regard the abutment structure as a rigid body and assume that the dynamic response of the system depends essentially on the surrounding soil.

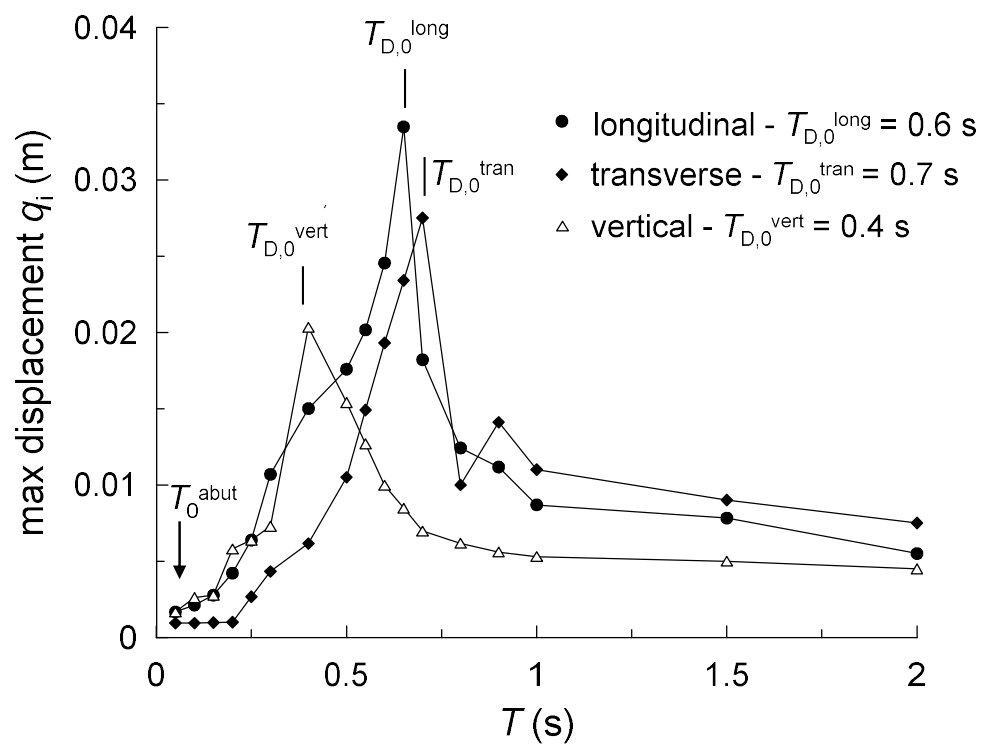

Figure 3: Dynamic amplification curves at small displacements of the soil-abutment system in the three translational degrees of freedom of the deck-abutment contact.

\subsection{Mass participation}

A first evaluation of the mass that participates to the dynamic response of the soilabutment system can be obtained from the dominant periods computed before. Thanks to the mono-modal shape of the amplification curves in Figure 3, as a first approximation the soilabutment system can be regarded as a single-degree-of-freedom system with resonance period $T_{\mathrm{D}, 0}{ }^{\mathrm{i}}$. The mass participation $m_{1}{ }^{\mathrm{i}}$ can be accordingly determined as follows:

$$
m_{1}^{\mathrm{i}}=\left(\frac{T_{\mathrm{D}, 0}^{\mathrm{i}}}{2 \times \pi}\right)^{2} \times K^{\mathrm{i}}
$$

with the superscript $i$ indicating the direction of motion and $K^{\mathrm{i}}=Q^{\mathrm{i}} / q^{\mathrm{i}}$ the static stiffness of the soil-abutment system, evaluated as the ratio between the amplitude of the force $Q^{\mathrm{i}}$ applied to the abutment top and the maximum displacement $q^{\mathrm{i}}$ occurring for large periods $(T \geq 2 \mathrm{~s})$. The resulting participating masses in the three directions of motion are listed in Table 2 . It is interesting to notice that the participating mass $m_{1}{ }^{i}$ results much greater than the sum of the mass of the abutment structure $\left(m_{\mathrm{abut}}=3.6 \times 10^{3} \mathrm{Mg}\right)$ and of backfill resting on the footing ( $\left.m_{\text {back }}=6.9 \times 10^{3} \mathrm{Mg}\right)$, named $m_{\text {abut }+ \text { back. }}$ In the horizontal directions the participating mass $m_{1}{ }^{\mathrm{i}}$ is about 3.5 times the mass of the structure and the soil fill, while in the vertical direction $m_{1}{ }^{i}$ is

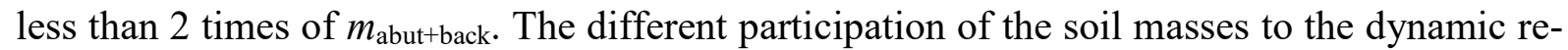
sponse of the abutment in different directions can be ascribed to the different deformation 
mechanisms that occur. In fact, in the longitudinal direction the main contribution to the whole response derives from the inertial effects transferred by the embankment, in the transverse direction the foundation soil and, to a minor extent, the embankment concur to the dynamic response of the structure, while in the vertical direction it can be assumed that soilabutment interaction is essentially controlled by the soil underneath the footing only.

\begin{tabular}{lcccc}
\hline direction & $T(\mathrm{~s})$ & $K^{1}(\mathrm{kN} / \mathrm{m})$ & $m_{1}{ }^{1}(\mathrm{Mg})$ & $\mathrm{m}^{1} / m_{\text {abut+back }}$ \\
\hline longitudinal & 0.6 & $5.2 \times 10^{6}$ & $4.7 \times 10^{4}$ & 3.39 \\
transverse & 0.7 & $4.1 \times 10^{6}$ & $5.1 \times 10^{4}$ & 3.63 \\
vertical & 0.4 & $6.4 \times 10^{6}$ & $2.6 \times 10^{4}$ & 1.84 \\
\hline
\end{tabular}

Table 2: Modal characteristics of the soil-abutment system in the longitudinal, transverse and vertical directions.

\section{CONCLUSIONS}

It has been shown that under dynamic conditions the stiffness of the constraint offered by abutment to the deck is markedly dependent on the frequency of the interaction forces acting on the abutment top, with a considerable increase of deformability in correspondence of the resonance of the soil-abutment system. For the abutment examined in this study, the dominant responses of the abutment occur in the range of periods $T=0.4 \div 0.7 \mathrm{~s}$ for the deformability of the foundation soil and, especially, because of the inertial effects that develop in the approach embankment. The mass participation depends on the direction of motion: it results to be about 3.5 times the mass of the abutment and the soil fill resting on the footing in the horizontal directions while it is about twice the latter in the vertical direction, demonstrating the involvement of a considerable part of the embankment and foundation soil in the dynamic response of the abutment.

\section{REFERENCES}

[1] C. Wissawapaisal, Modelling the seismic response of short bridges. Thesis, University of Illinois at Urbana, Champaign (IL), 1999.

[2] A. Kotsoglou, S. Pantazopoulou, Bridge-embankment interaction under transverse ground excitation. Earthq Eng Struct Dyn, 36(12), 1719-1740, 2007.

[3] D.N. Gorini, L. Callisto, A coupled study of soil-abutment-superstructure interaction. Submitted to Proc. of the Italian National Congress of Geotechnical Researchers (CNRIG2019) entitled "The Geotechnical Research for the Protection and Development of the Territory", (July 3-5, 2019).

[4] F. McKenna, Object-oriented finite element analysis: Frameworks for analysis, algorithms and parallel computing. Ph.D. dissertation, Univ. of California, Berkeley, CA, 1997.

[5] F. McKenna, G.L. Fenves, M.H. Scott, B. Jeremic, Open system for earthquake engineering simulation. http://opensees.berkeley.edu, 2000.

[6] N.D. Diaz, P.S. Amat, GID the personal pre/postprocessor user's manual, version 5.0. CIMNE, Barcelona, Spain, http://gid.cimne.upc.es, 1999. 
[7] V. Fioravante, D. Giretti, M. Jamiolkowski, G.F. Rocchi, Triaxial tests on undisturbed samples of gravelly soils from the Sicilian shore of Messina strait. Bulletin of Earthquake Engineering, 10, 1717-1744, 2012.

[8] M. Jamiolkowski, D.C.F. Lo Presti, Geotechnical characterization of Holocene and Pleistocene Messina sand and gravel deposits. Charact. and Engng. Properties of Natural Soils, Singapore, Balkema Publishers, 2, 1087-11120, 2002.

[9] E.N. Dvorkin, K.J. Bathe, A continuum mechanics based four node shell element for general nonlinear analysis. Eng. Comput., 1, 77-88, 1984.

[10] Z. Yang, A. Elgamal, E. Parra, A computational model for liquefaction and associated shear deformation. J. Geotech. Geoenviron. Eng., 129(12), 1119-1127, 2003.

[11] D.N. Gorini, Soil-structure interaction for bridge abutments: two complementary macro-elements. PhD thesis, Sapienza University of Rome, Rome, Italy. 2019.

[12] F. McKenna, G.L. Fenves, Using the OpenSees interpreter on parallel computers, Network for Earthquake Engineering Simulations. Berkeley, California (US), 2008. 